\title{
Analysing system behaviour by automatic benchmarking of system-level provenance
}

\author{
Middleware 2019 Doctoral Symposium
}

\author{
Sheung Chi Chan \\ University of Edinburgh \\ United Kingdom \\ s1536869@inf.ed.ac.uk
}

\begin{abstract}
System-level provenance is of widespread interest for applications such as security enforcement and information protection. However, testing the correctness or completeness of provenance systems is challenging. In some cases, there is not even a clear consensus about what behaviour is correct. This work presents the study of system behaviour through the analysis of system-level provenance and the provenance systems that collect them. Besides, an automated tool, ProvMark is presented for the automation of the process and provides an additional layer of expressiveness benchmarking on existing provenance systems and their provenance result. This helps to understand patterns of system behaviour for security and other applications. It also allows provenance system developers to verify their tools and allows end-users to compare the tools at the same level to choose a suitable one for their purposes.
\end{abstract}

CCS Concepts - Applied computing $\rightarrow$ System forensics; • Software and its engineering $\rightarrow$ Operating systems; Software verification and validation; • Computing methodologies $\rightarrow$ Logic programming and answer set programming.

Keywords provenance, benchmarking, subgraph isomorphism

ACM Reference Format:

Sheung Chi Chan. 2019. Analysing system behaviour by automatic benchmarking of system-level provenance: Middleware 2019 Doctoral Symposium. In Middleware '19: 20th International Middleware Conference Doctoral Symposium (Middleware '19), December 9-13, 2019, Davis, CA, USA. ACM, New York, NY, USA, 5 pages. https://doi.org/10.1145/3366624.3368155

\section{Introduction}

Data provenance is increasingly used for security, through forensic audit [6] or online dynamic detection of malicious behaviour [2, 3]. To cater to the requirements of different use-cases, there are many system-level provenance capture systems in the literature covering a variety of operating systems from Linux and BSD to Android and Windows. Each system assembles low-level system events into a high-level provenance graph describing processes, system resources, and causal relationships among them.

Often, such systems are described as capturing a complete and accurate description of system activity. To date, the designers of

Permission to make digital or hard copies of all or part of this work for personal or classroom use is granted without fee provided that copies are not made or distributed for profit or commercial advantage and that copies bear this notice and the full citation on the first page. Copyrights for components of this work owned by others than the author(s) must be honored. Abstracting with credit is permitted. To copy otherwise, or republish, to post on servers or to redistribute to lists, requires prior specific permission and/or a fee. Request permissions from permissions@acm.org.

Middleware '19, December 9-13, 2019, Davis, CA, USA

(C) 2019 Copyright held by the owner/author(s). Publication rights licensed to ACM.

ACM ISBN 978-1-4503-7039-4/19/12...\$15.00

https://doi.org/10.1145/3366624.3368155 each such system have decided how to interpret these goals independently, making different choices regarding what activity to record and how to represent it. Although some of these systems do use standards such as $\mathrm{W} 3 \mathrm{C} \mathrm{PROV}^{1}$ that establish a common vocabulary for provenance-related data, such standards do not specify how to record operating-system-level behaviour, or even when such records are considered "accurate" or "complete". Indeed, as we shall see, in practice there is little consensus about how specific activities (e.g., renaming a file) should be represented in a provenance graph. Additionally, different systems also work at different system layers (e.g., kernel space vs. userspace), so some information may be unavailable to a given system.

Security analysts, auditors, or regulators seeking to use these systems face many challenges. Some of these challenges stem from the inherent size and complexity of provenance graphs: it is not unusual for a single day's activity to generate graphs with millions of nodes and edges. However, if important information is missing, then these blind spots may render the records useless for their intended purpose. We advocate a pragmatic approach as a first step toward the goal of validating and testing provenance systems, which we call expressiveness benchmarking. The motivation and goal of such benchmarking are to answer questions such as:

- What does each node/edge in the graph tell us about actual events? (correctness, accuracy)

- What information is guaranteed to be captured and what are the blind spots? (completeness)

The main contributions are as follows:

- Providing ways to analyse the capability of provenance systems in different applications

- Providing ways to analyse provenance describing systemlevel behaviour

- Providing ways to solve graph matching problems with lightweight implementation, based on answer set programming.

\section{Use Cases}

To clarify when the results of the expressiveness benchmarking are useful, we outline several common use cases. These use cases can target general end-users and provenance system developers.

Tracking failed calls Show provenance systems that track failed system calls.

Configuration validations Validate policies/configurations.

Regression testing Compare snapshots to discover unexpected alteration.

Suspicious activity detection Identify activity patterns and indicate the existence of the activities.

${ }^{1}$ https://www.w3.org/TR/prov-overview/ 


\section{Manual Expressiveness Benchmarking}

The pragmatic approach of expressiveness benchmarking is inspired by previous community efforts such as the Provenance Challenge exercises [5]. Our work proposes an "expressiveness benchmark" for OS-level provenance recording systems. The goal of such a benchmark is not to produce an absolute ranking of systems, but rather to help place different systems in a landscape so that their relative capabilities can be better understood. We hope that the process of benchmarking and comparing provenance recording systems on comparable examples will help lead to greater uniformity and semantic interoperability among such systems, or greater understanding of design decisions.

The goal of expressiveness benchmarking is to identify the provenance patterns for the smallest meaningful units: system calls operating on the OS kernel. These system calls combine in different permutations to form high-level processes. To understand what system calls have been involved in the processes and their provenance patterns, we need to understand how each system call contributes to the resulting provenance graph. That is, we need to understand the explicit patterns of provenance for each system call. This mapping provides a benchmark for each system call and its effect on the resulting provenance graph. With this understanding, we can use the patterns to generate rules to identify the existence of highlevel actions (containing different permutations of system calls) in a large system by analysing the provenance graph describing its runtime behaviour. This can help to trace the existence and initiator of certain actions for security and forensic analysis.

Before considering how the system-call patterns can help in the process, we aim to develop some building blocks manually for proof of concept. In this first stage of work, we carried out benchmarking manually by running them with suitable input on two provenance collecting tools and observing the provenance information generated by them. We also performed some manual identification, classification and comparison to retrieve the provenance patterns for those system-calls, generated by different tools. This manual work helped us to build up and consolidate the foundational work, which provides a basic understanding of how those system-calls are displayed as provenance patterns. It also helps to demonstrate the feasibility of this study on a bigger scale. This manual work aims to provide qualitative answers to demonstrate the non-unified standard of different provenance tools and the feasibility of our expressiveness benchmarking approach.

Although it is possible to do the benchmarking manually, it may be error-prone and needs a lot of human effort to do so. We understand from the manual experiments, a simple provenance graph representing an empty binary execution already has around 40 nodes and edges, together with $100+$ property values. This problem will become more serious if we move to handle real-world program executions which consist of a large set of system calls. If we continue to use manual effort for handling the increasing size of provenance graphs from real-world programs, the error rate and the resources needed will also be increased. That is why we aim to develop a fully automated system, ProvMark, for handling the whole benchmarking process. This should benefit both the provenance recording tools' developers in understanding, cross-checking and enhancing their tools and the end-users who can use ProvMark to research which tools are best suited for their use case scenario.
The proposed expressiveness benchmarking approach together with the summary of the manual experiments has been presented in the 9th USENIX Workshop on the Theory and Practice of Provenance (Published Work \#1).

\section{ProvMark System}

From the need to automate the expressiveness benchmarking, we build up the automated tool, ProvMark. The design and evaluation of ProvMark described in Section 4 and 5 has been presented in the 20th International Middleware Conference (Published Work \#2).

ProvMark is intended to automatically identify the subgraph of a provenance graph that is recorded for given target activity. Target activities could consist of individual system calls (or syscalls), sequences of syscalls, or more general (e.g., concurrent) processes. For the moment, we consider the simplest case of a single syscall, but the same techniques generalize to sequential target activities.

We call the targeted system call the target call and the corresponding subgraph the target graph. Naively, one might proceed by writing a simple $C$ program for each target system call that just performs that call and nothing else. However, starting and ending a process creates considerable "boilerplate" provenance, including calls as well as accesses to libraries and, sometimes, memory mappings. Furthermore, some target calls require other prerequisite calls to be performed first. For example, analysing a read or close system call requires first performing an open. Process startup and prerequisite calls are both examples of background activity that we would like to elide.

In each benchmark, we use a \#ifdef TARGET CPP directive to identify the target behaviour of interest. ProvMark generates two executables for each such benchmark: a foreground program that includes all code in the benchmark program, and a background program that contains the background activities only. The two binaries are almost identical; the difference between the resulting graphs should precisely capture the target behaviour.

ProvMark is composed of four subsystems: (1) recording, (2) transformation, (3) generalization, and (4) comparison. Users can select which provenance capture system (SPADE, OPUS and CamFlow are currently supported) to use, which benchmark to run, and other configuration settings, such as the number of trials.

\subsection{Recording}

The recording subsystem runs the provenance capture tools on test programs. This subsystem first prepares a staging directory that provides a consistent environment for test execution. The recording subsystem then starts the provenance capture tool with appropriate settings, captures the provenance generated by the tool, and stops the tool afterwards.

The recording subsystem is used to record provenance graphs for the foreground and background variants of each program. Provenance can include data that varies across runs, such as timestamps, so we typically record multiple runs of each program and filter out the transient information in a later generalization stage.

\subsection{Transformation}

Different systems output their provenance graphs in different formats, including Graphviz DOT format, Neo4J storage, W3C PROVJSON [4] as well as some other storage or stream processing backends. To streamline the remaining stages, we translate these three 
formats to a common representation, Datalog, which is a Prolog-like logical language used by Answer Set Programming.

All the remaining stages, including the final result, work on the Datalog graph representation, so these stages are independent of the provenance capture tool and its output format. The Datalog representation can easily be visualized.

\subsection{Graph Generalization}

The third subsystem performs graph generalization. We wish to identify a single, representative and general graph for each test program. To formalize these notions, we adapt the notion of graph isomorphism to property graphs. This subsystem uses an answer set programming solving collection, Potassco ${ }^{2}$ to help solve the hard combinatorial search problem of graph isomorphism.

We assume that over sufficiently many recording trials, there will be at least two representative ones, which are similar to each other. Identifying two representative runs is complicated by the fact that there might be multiple pairs of similar graphs. To obtain two representative graphs, we first consider all of the trial runs and partition them into similarity classes. We first discard all graphs that are only similar to themselves and consider these to be failed runs. Among the remaining similarity classes, we choose a pair of graphs whose size is smallest. This pair forms the base graphs for the generalization process.

Given two similar graphs, the generalization stage identifies the property values that are consistent across the two graphs and removes the transient ones. The generalization stage searches for matching of nodes and edges of the two graphs that minimize the number of unmatched properties. We assume that the differences are transient data and discard them. We would like to match as many properties as possible.

\subsection{Graph Comparison}

The fourth and last subsystem is graph comparison. Its purpose is to match the background graph to a subgraph of the foreground graph; the unmatched part of the foreground graph corresponds to the target activity.

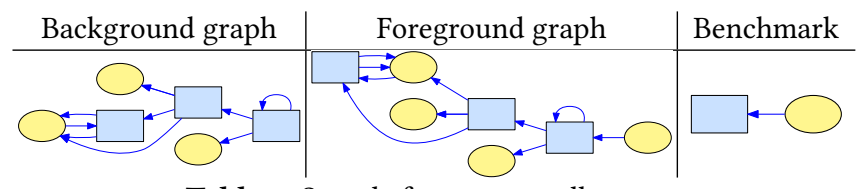

Table 1. Sample for system call creat

Because provenance recording is generally monotonic (i.e. only appending), we expect that the background provenance graph is a subgraph of the foreground provenance graph, so there should be a one-to-one matching from the nodes and edges in the background graph to the foreground graph. This graph matching problem is a variant of the subgraph isomorphism problem, a classical NP-complete problem [1]. We again solve these problems using Potassco $^{2}$, taking advantage of the fact that it can search for optimal solutions according to some cost model. A sample background graph, foreground graph and resulting benchmark for system call creat are shown in Table 1, where the property labels are ignored

\footnotetext{
${ }^{2}$ https://potassco.org/
}

due to space limits. In those benchmarks, the blue rectangles represent processes, while the yellow ovals represent artefacts in the system, such as files.

\section{Sample Result}

There are some sample benchmarks shown in Table 2 . These benchmarks (property labels omitted) are generated by ProvMark using one of the provenance systems on three system calls. The green ovals represent dummy nodes, which exist to keep the graph intact.

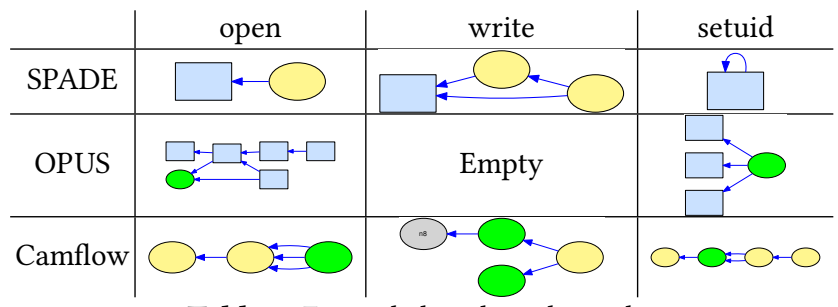

Table 2. Example benchmark results

\section{Non-determinism}

Nowadays, non-determinism is quite common in computing. For example, operation systems rely on concurrent execution to increase performance and efficiency, and distributed computing rely on asynchronous network communication to avoid blocking of resources. Thus, it is hard to avoid the non-deterministic characteristics when we try to analyse behaviours of system activities. One important motivation for the research is to provide ways to benchmark and analyse real system behaviour for security usage. This can aid the developers and end-users of provenance systems in finding problems or choosing suitable tools for their uses. In these real-world applications of ProvMark, the provenance benchmarking system cannot avoid the handling of non-deterministic input. Thus this extension of ProvMark has a high priority.

The handling of non-deterministic input includes multiple steps, the first thing we need to do is to identify which of the graphs represent the same schedules of non-deterministic events. As we notice, if two trial runs follow the same schedule, then they will have the same system calls and execution order. Thus we can identify them by matching their system call order list. This could be retrieved by monitoring the Linux Security Enhancement (Linux $\mathrm{SE}$ ) in the kernel as each of the system call execution needs to go through Linux SE for permission checking before execution. To decrease dependencies from writing customized kernel modules to do the monitoring directly, we instead choose to use an existing kernel framework Ftrace to handle the monitoring work. Ftrace is a framework shipped with the Linux kernel, aiming to monitor and trace functions and activities at the kernel level. There is also a tool named trace-cmd that allows users to configure Ftrace from user level and it will also pass back the result of Ftrace from the kernel to user level. With this understanding, in each trial run of the foreground program, we use trace-cmd to start Ftrace alongside the provenance collecting tools to capture the list of system calls for the execution of the foreground program. We also configure the provenance collecting tools to ignore the system calls generated by the process that controls trace-cmd and any of its child processes to avoid additional provenance from the trace-cmd utility and the Ftrace framework. After each trial run, we get a system call schedule corresponding to each foreground provenance graphs. 
To limit the size of the comparator and to have a common format of fingerprints, we create a hash for each of the schedules. We first generate a long string by concatenating all the system call names in order. After that, we create a hash for this string to form the fingerprint. The fingerprint is guaranteed to have fixed size and format. It is used directly as the identifier for the attached graph. The next step is to group the foreground graphs. Graphs with the same fingerprint will be grouped because it means that they executed the same system calls in the same order assuming no hash collision occurs. After the grouping of the graphs, each group is passed on to the generalization subsystem separately to continue the process, the fingerprint for each group should also be preserved. As a result, each group will produce a generalized graph separately.

The clear difference from the handling of deterministic events is that there are multiple groups of graphs that need to go through the generalization and comparison process. In deterministic input, we assume that all graphs should be similar to each other. This assumption allows the graphs to be generalized directly. After that, the generalized background and foreground graph are compared to each other to retrieve the provenance benchmark. In the case of nondeterministic input, there are multiple sets of foreground graphs, only the graphs in each set are similar to each other. Thus only the graphs in the same set will be generalized together. As a result, there will also be multiple generalized foreground graphs. Each generalized foreground graph is associated with a unique group fingerprint and is compared to the background graph to retrieve a provenance benchmark pattern. At last, ProvMark generates a set of provenance benchmark graphs after processing non-deterministic program input and each of the provenance benchmark results are labelled by their fingerprint.

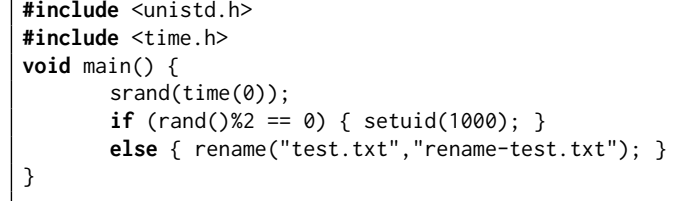

Code Snippet 1. Example for non-determinism

Code Snippet 1 contains a sample non-deterministic input. This program will execute either of the two system-calls setuid and rename depending on the random seed. This results in two different schedules and ProvMark will group the graphs accordingly and apply generalization and comparison separately. As a result, two separate benchmarks representing each schedule are generated, which are shown in Table 3 (property labels omitted).

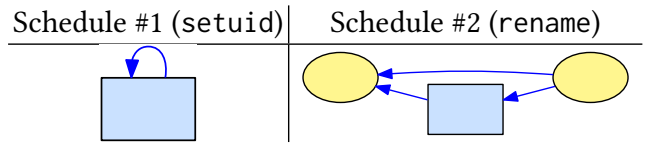

Table 3. Sample benchmark for Code Snippet 1

\section{Discussion}

Although ProvMark already provides some level of automation of the proposed expressiveness benchmarking approach, there are still some limitations and possible improvements.

In the current development of ProvMark, we limited the testing and application to provenance systems running under Linux. It is believed but not tested that ProvMark should be adaptable to other operating systems and working environments. Besides, the current design of ProvMark still requires manual effort for configuration and comparison of the resulting provenance benchmark patterns. These manual efforts require an understanding of system behaviour and provenance structure. Although ProvMark is capable of handling benchmark programs with a small number of system calls, scaling up to larger amounts of target activity appears challenging. This is because of the NP-completeness and worst-case exponential behaviour of subgraph isomorphism testing required in the generalization and graph comparison stage. This problem becomes more serious when handling non-deterministic input as there are more generalization and comparison steps required as each schedule in non-deterministic input needs to be handled separately.

There are several directions for future work, to address the limitations stated above. First, additional support for automating the process of creating new benchmarks, or interpreting the results would increase the usefulness of the system, as would extending it to provenance tracking at other levels, such as distributed system coordination layers. An ideal case would be providing an abstract layer on top of ProvMark to automatically return a suggestion for end-users in choosing a provenance system for certain applications, or to automatically point out possible problems for provenance system developers in regression testing results. Also, the automated approach should be tested or extended to handle realistic applications such as suspicious behaviour analysis which would require dealing with much larger target activities and graphs.

\section{Conclusions}

This work presents the expressiveness benchmarking on provenance systems and their generated provenance. To the best of our knowledge, it is the first work to address the unique challenges of testing and comparing provenance systems. The manual foundations and the need for an automated system are discussed at the beginning, then the design of ProvMark the automated system is outlined. Lastly, some result demonstration and future improvements of ProvMark are discussed. In conclusion, ProvMark provides automation of the proposed expressiveness benchmarking approach which is a significant step towards validation of provenance systems and should be useful for developing unified correctness or completeness criteria for them.

\section{Published Work}

1. Chan, S. C., Gehani, A., Cheney, J., Sohan, R., \& Irshad, H. (2017). Expressiveness benchmarking for system-level provenance. In Proceedings of the 9th USENIX Workshop on the Theory and Practice of Provenance. USENIX, 2017.

2. Chan, S. C., Cheney, J., Bhatotia, P., Pasquier, T., Gehani, A., Irshad, H., Carata, L., \& Seltzer, M. (2019). ProvMark: A Provenance Expressiveness Benchmarking System. In Proceedings of the 20th International Middleware Conference. ACM, 2019.

\section{Acknowledgments}

This material is based upon work supported by the Defense Advanced Research Projects Agency (DARPA) under contract FA8650-15-C-7557 and sponsored by the Air Force Office of Scientific Research, Air Force Material Command, USAF, under grant number FA8655-13-1-3006. The U.S. Government and University of Edinburgh are authorised to reproduce and distribute reprints for their purposes notwithstanding any copyright notation thereon.

Thanks go to James Cheney and Pramod Bhatotia for their supervision, long term support and insightful comments on the work. Second thanks go to the co-authors of the published papers for their support and comments on the work. 


\section{References}

[1] Stephen A. Cook. 1971. The Complexity of Theorem-proving Procedures. In Proceedings of the Third Annual ACM Symposium on Theory of Computing (STOC '71). ACM, New York, NY, USA, 151-158.

[2] Xueyuan Han, Thomas Pasquier, Tanvi Ranjan, Mark Goldstein, Margo Seltzer and PDF Han. 2017. FRAPpuccino: Fault-detection through Runtime Analysis of Provenance. In Proceedings of the 9th USENIX Workshop on Hot Topics in Cloud Computing (HotCloud'17). USENIX Association.

[3] Wajih Ul Hassan, Mark Lemay, Nuraini Aguse, Adam Bates, and Thomas Moyer 2018. Towards Scalable Cluster Auditing through Grammatical Inference over
Provenance Graphs. In Proceedings of the 25th Annual Network and Distributed System Security Symposium (NDSS 2018).

[4] Trung Dong Huynh, Michael O. Jewell, Amir Sezavar Keshavarz, Sanius T. Michaelides, Huanjia Yang, and Luc Moreau. 2013. The PROV-7SON Serialization. Technical Report. World Wide Web Consortium. https://www.w3.org/ Submission/2013/SUBM-prov-json-20130424/

[5] Luc Moreau et al. 2008. Special Issue: The First Provenance Challenge. Concurrency and Computation: Practice and Experience 20, 5 (2008), 409-418.

[6] Wei Wang and Thomas E. Daniels. 2008. A Graph Based Approach Toward Network Forensics Analysis. ACM Trans. Inf. Syst. Secur. 12, 1, Article 4 (Oct. 2008), 33 pages. 